\title{
Analyzing pixel spread correlation with lenticular lens efficiency on multi user VR displays
}

\author{
Juan Sebastian Munoz-Arango \\ Emerging Analytics Center \\ University of Arkansas Little Rock \\ 72204, Little Rock, AR, USA \\ jsmunozaran@ualr.edu
}

\author{
Dirk Reiners \\ Emerging Analytics Center \\ University of Arkansas Little Rock \\ 72204, Little Rock, AR, USA \\ dpreiners@ualr.edu
}

\author{
Carolina Cruz-Neira \\ Emerging Analytics Center \\ University of Arkansas Little Rock \\ 72204, Little Rock, AR, USA \\ cxcruz@ualr.edu
}

\begin{abstract}
One of the all time issues with Virtual Reality systems regardless if they are head-mounted or projection based is that they can only provide perspective correctness to one user. Such limitation affects collaborative work which is nowadays the standard anywhere. One of the approaches for generating different perspective correct images to several users is through optical routing. This approach relies on bending the light to generate perspective correct images to the engaged users. Lenticular lenses can bend the light to be able to generate perspective correct images for several users depending on their positions. On this paper we present an analysis that lets us understand the pixel spread correlation with lenticular lens efficiency on multi user VR displays.
\end{abstract}

\section{Keywords}

Multi user displays, Virtual Reality, Simulation, lenticular lens

\section{INTRODUCTION}

Different approaches have been proposed across the years for interacting through Virtual Reality (VR), head mounted displays (HMDs), projection-based virtual reality systems, volumetric screens and several different devices aid on these tasks.

When the person who is interacting in VR is by himself, HMDs work well to some extent. Unfortunately, working alone is rarely the case nowadays in any field. Several attempts have been proposed to work cooperatively with HMDs [1] but unfortunately having weight on one's head not only exhausts users but also hinder their eyesight when used on prolonged sessions. Although some sessions can be short, another issue that raises is that HMDs occlude one's face, hence making facial expressions less visible to other participants removing important nonverbal communication channels.

Normally, when a group of experts get together, they discuss and gesture around a common dataset hoping to achieve a consensus. This engagement is persistent across disciplines and begs for a VR system that accomodates small groups of people working together with correct perspective point of views. Different studies [2] [3] back up the perspective correctness requirement demonstrating that collaboration times get significantly longer when participants dont have a correct perspective point of view compared to a correct one.

An interesting classification for different approaches when multiplexing images from a single display was presented by Mark bolas et al in [4]. Here, they introduce a "solution framework" of four categories for multiplexing images: Spatial Barriers, Optical Filtering, Optical Routing and Time Multiplexing.

Lenticular lenses fall in the optical routing approach and are widely used for lenticular printing, 3D TV and lenticular screens among other uses [5][6][7]. These lenses can also used for multiplexing views from a single screen. It has been shown that the lens specs that maximizes the minimum visible unique pixels and minimizes ghosting is a lenticular lens that has a $50 \%$ radius increase from the minimum possible radius, covers 18 to 21 pixels per lenticule and has the smallest possible substrate thickness in [8].

Pixel spread is also an important factor that also contributes to ghosting between users. On this paper we are going to present and discuss the correlation factor of pixel spread with lenticular lens performance for multi user displays.

\section{BACKGROUND}

\subsection{Lenticular Lens Overview}

A lenticular lens can be thought as a set of cylinders (lenticules) placed next to each other overlapping with one side flat and the other side with the protubing lenses (fig 1), these lenses are designed so that when viewed from different angles different slices of an image are displayed (fig 2).

Lenticular printing is one of the most prominent examples of lenticular lens usage. Here, lenses are employed to give illusion of depth or to make images that change depending on the angle the print is looked from. 


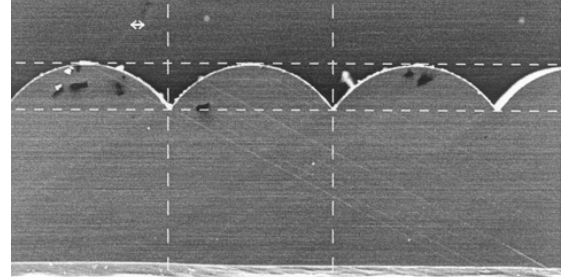

Figure 1: Micro profile of a lenticular lens.

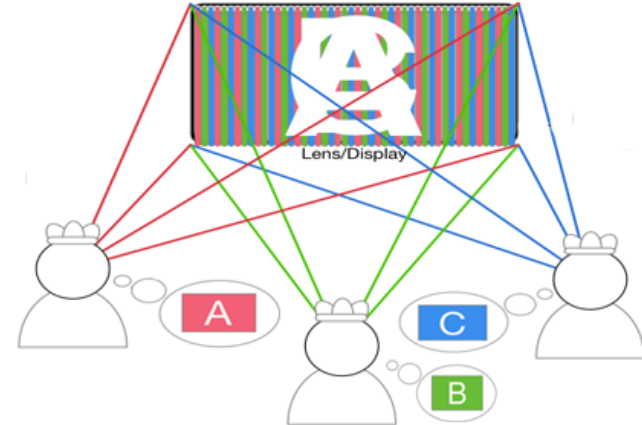

Figure 2: Image looked from different angles in a lenticular lens

Depending on how pronounced the lenticules are engraved in the sheet, different types of effects can be achieved (fig 3). These effects can be just about anything one can do with video; some of these effects include morphing effects, animations, flipping and 3D effects [9] [10].

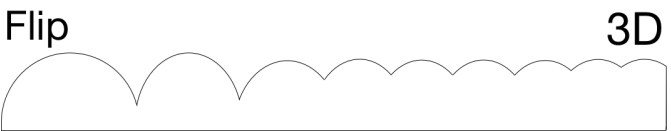

Figure 3: Flip to 3D lenticular lenses.

- Morph effect: This is commonly used to create the illusion of transformation (fig. 4 A).

- Animation effect: Generates illusion of motion from a set of sequential images (fig. 4 B)

- Flip effect: A dramatic swap of two images occurs vanishing and then reappearing from one to another (fig. 4 C).

- 3D effect: Provides an illusion of depth and perspective by layering objects within images (fig. 4D).

Lenticular lenses are classified by the number of lenticules that can be fit in an inch (Lenses per inch (LPI)), the higher the LPI the smaller each lenticule is. Manufacturers normally offer lenses in 10, 15, 20, 30, 50, $60,75,100 \mathrm{LPI}$ lenses; however, with enough funds one can get a custom lens manufactured with specific characteristics.

Following the same principle of how lenticular printing works, lenticular lenses can also be used for multiplexing different images to different users in VR under an optical routing approach.

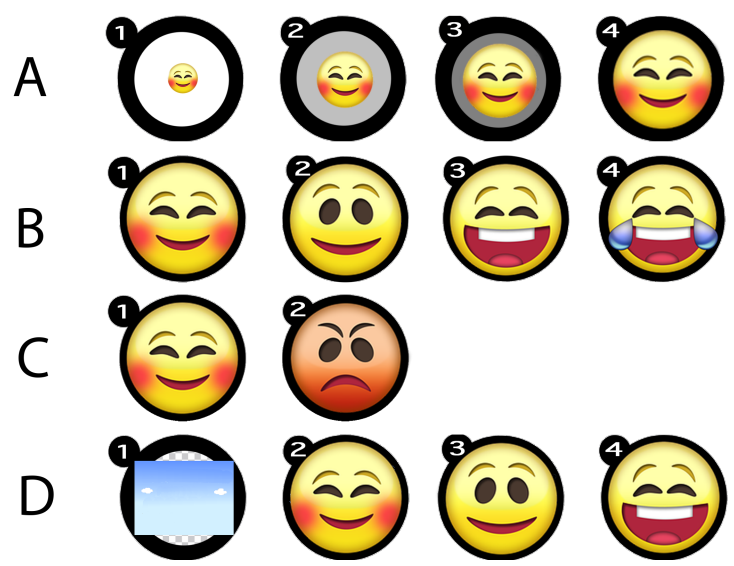

Figure 4: Effects. A:Morph B:Animation C:Flip D:3D

\subsection{Pixel overview}

Pixels are the building blocks of screens; pixels are composed of subpixels which are generally colored red green and blue; When all the three subpixels are at full intensity a white pixel light is generated; when all the subpixels are completely dark; black is the resulting color; combination of intensities of the different subpixels generate colors like pink, yellow, etc.

An important factor that occurs on screens is pixel spread. Pixel spread is referred in this paper as the angle from where the light can be seen from each pixel. Naturally, for TVs, monitors and screens in general, an increased pixel spread is the desired effect so people can see the same image from different angles without any variation in contrast / brightness.

Pixel spread for the purpose of this paper varies from 0 (collimated light) to 89 degrees (fan like setting). The more pixel spread the wider the angle an image on a screen can be seen from (fig. 5).

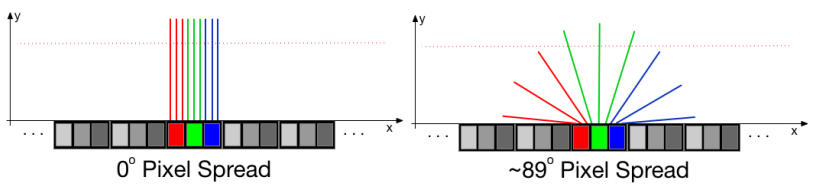

Figure 5: Pixel spread range

\section{PREVIOUS WORK}

Optical routing uses the angle-sensitive optical characteristics of certain materials to direct or occlude images based on the user's positionm [4].

In 1994, Little et al talk about a design for an autostereoscopic, multiperspective raster-filled display [11]. Here, they propose a time multiplexed approach and an optical routing approach. The optical routing approach features video cameras and LCTV projectors. Here, they use an array of video cameras to capture multiple perspective views of the scene and then they fed these to an array of LCTVs and simultaneously 
project the images to a special pupil-forming viewing screen. The viewing screen is fabricated by either a holographic optical element or a Fresnel lens and a pair of crossed lenticular arrays.

Van Berkel et al in [12] [13] built a prototype display using a LCD and a lenticular lens from Philips Optics to display 3D images; they slanted the lenticular lens with respect to the LCD panel in order to reduce the "picket fence" effect.

Later in the same year, Matsumoto et al in [14] proposes a system that consists of combination of cylindrical lenses with different focal lengths, a diffuser screen and several projectors to create a $3 \mathrm{D}$ image. They had issues with one of the lenses causing a dark stripe in the $3 \mathrm{D}$ image affecting the stereoscopic vision by reducing the sense of depth.

Omura, presents a system that uses double lenticular lenses with moving projectors that move according to the tracked user's position to extend the viewable area [15], their system needs a pair of projectors per person and their projectors move to adjust each user's position. Their system suffers from latency due to the mechanical movement.

Lipton proposed the Synthagram [16], a system that consists of an LCD Screen with a lenticular screen that overlays the LCD display. They angled the lenticular screen in order to reduce moire patterns and their system uses nine progressive perspective views from a single image. They sample these views into a program called the Interzig where they process the images and assign each pixel to a specific position in the screen.

Matusik proposes a system that consists of an array of cameras, clusters of network connected PCs and a multi-projector 3D display with the purpose to transmit autostereoscopic realistic 3D TV [17]. They record the imagery with a small cluster of cameras that are connected to PCs. The PCs broadcast the recorded video which later on is decoded by another cluster of consumer PCs and projectors. Their 3D Display consists of 16 NEC LT-170 projectors that are used for front or rear projection. The rear projection approach consists for two lenticular sheets mounted back to back with an optical diffuser material in the center and the front projection system uses one lenticular sheet with a retro reflective front projection screen material.

Another way of optical routing approach use is the display proposed by Nguyen et al [18] [19]. Here, they propose a special display which consists of a screen with 3 layers that has directional reflections for projectors so each participant sees a customized image from their perspective; their system supports up to 5 viewing zones but doesn't support tracking and it needs a projector per participant.

Takaki et al introduces a system that can produce 72 views[20]. Their system consists of a light source ar- ray, a micro lens and a vertical diffuser (a lenticular sheet). They mention that as the horizontal positions of all light sources are different, rays from different light sources proceed to different horizontal directions after passing through the micro lenses thus generating different views. They also mention that it's difficult to fabricate a large micro lens array and also say that unused pixels remain at the corners of the LCD panel.

Later on. In [21] [22] followed by [23], Takaki discusses a multiple projection system that is modified to work as a super multiview display. Here, they attach a lens to the display screen of a HDD projector and by combining the screen lens and the common lens, they project an aperture array. This aperture array is placed on the focal plane of the common lens, and the display screen (a vertical diffuser) is placed on the other focal plane. Hence, the image of the aperture array is produced on the focal plane of the screen lens. With this, the image of an aperture array gets enlarged generating enlarged images that become viewpoints. The authors comment that there is some discontinuity between the different generated views when the observation distance is different from the distance to the viewpoints.

In 2009. Takaki and his team introduce a prototype panel that can produce 16 views [24]. They do this by building a LCD with slanted subpixels and a lenticular screen. They place a diffusion material between the lenticular sheet and the LCD screen in order to defocus the moire pattern but increase the crosstalk among viewpoints. They mention that by slanting the subpixel arrangement instead of the lenticular sheet, they can increase the number of views but the optical transmittance of the display decreases. They conclude that by slanting the subpixels in the screen instead in the lenticular sheet, they can reduce significantly the crosstalk and moire compared to the normal approaches. Their approach requires to build a LCD display which is a quite a complex task.

Finally, in 2010 Takaki and his team combine several 16-view flat-panels that have slanted subpixels[24] and creates a system with 256 views[25]. They superimpose the different projected output of the panels to a single vertical diffuser. The multiple viewing zones for each flat panel are generated on an incident pupil plane of its corresponding projection lens. Each projection lens projects to the display surface of its corresponding flat panel system on the common screen and finally a screen lens is located on the common screen so the lens generates viewing zones for observers. They mention that their prototype display has the possibility of producing $3 \mathrm{D}$ images on which the human eye can focus but also they report that there is considerable crosstalk between the viewing zones and the resolution of the prototype is not very high. 
Another system that takes advantage of the optical routing approach is the Free2C display, a project proposed by Surman in [26]. Here, they created a single viewer autostereoscopic display using a head tracker. The display accommodates the head movement of the viewer by continually re-adjusting the position of the lenticular lens in relation to the LCD to steer the stereoscopic views onto the eyes of the viewer. Their display resolution is $1200 \times 1600$, the viewing distance goes from $40 \mathrm{~cm}$ to $110 \mathrm{~cm}$ and side to side movements range of approximately +-25 degrees from the center of the screen. They also attempted a multi-user display that steers the LCD instead of the lenses to produce image regions for the users but they mention the display performance was really poor.

Similarly to Free2C, Brar et al use image recognition to track users' heads to produce multiple steerable exit pupils for left and right eyes [27] [28]. Here, they describe the design and construction of a stereoscopic display that doesnt require wearing special eye wear. A stereo par is produced on a single LCD by simultaneously displaying left and right images on alternate rows of pixels. They propose steering optics controlled by the output the aforementioned head tracker to direct regions, referred as exit pupils to the appropriate viewers' eyes. Their prototype is not optimal due to insufficient brightness and instability in the holographic projector and their current research doesn't support multiple users.

Kooima et al [29], uses 24" and 42" 3DHD Alioscopy displays[30] which come with integrated lenticular lenses. They propose a system that consists of scalable tiled displays for large field of views and use a generalization of a GPU based autostereoscopic algorithm for rendering in lenticular barriers. They tried different methods for rendering but they had issues where they perceived repeated discontinuities, exaggerated perspectives and as the displays pixels cannot be moved smoothly but in discrete steps. The tracked viewer moves into transition between channels, the user begins to see the adjacent view before the channel perspective is updated to follow the user's head.

Zang et al proposes a frontal multi-projection autostereoscopic display [31]. Their approach consists of 8 staggered projectors and a 3D image guided screen. The 3D image screen is mainly composed of a single lenticular sheet, a retro-reflective diffusion screen and a transparent layer that is filled between them to control the pitch of the rearranged pixel stripe in interlaced images. Their system is space efficient compared to previous approaches that produce light from the back of the screen, but the loss of intensity and crosstalk are seriously increased out of the system boundaries and besides being complex it doesn't provide perspective perfect views for each user.
We have mentioned here some research that has been done throughout the years that use an optical routing approach; specifically, lenticular lenses to separate users. Still, none of these projects compare the pixel spread correlation with the performance of said lenticular lenses.

\section{MATHEMATICAL MODEL}

Depending on the lens / screen marriage used, different lens parameters need to be set for the best performance. Some factors like the minimum number of pixels perceived, ghosting, color banding among others can be improved / minimized depending on the values the lens have. Such values need to be taken into account when pursuing an optical routing approach for multiplexing images.

To know how good or bad a specific lens with specific attributes works with any given screen, a lenticular lens simulator was developed. Such simulator helped determine the best parameters for a lens given a specific screen. This simulator also can assess how the screen's pixel spread is correlated with the lens performance.

Lenticular lenses have different parameters that can be tweaked to produce different effects; manufacturers sell lenses based on their LPI but at the end, each of these lenses comes with a set of specifications like the refraction index of the material the lens is made of, substrate thickness, viewing angle, lenticule radius, etc.(fig. 6). In order to simulate how a lenticular lens works, we

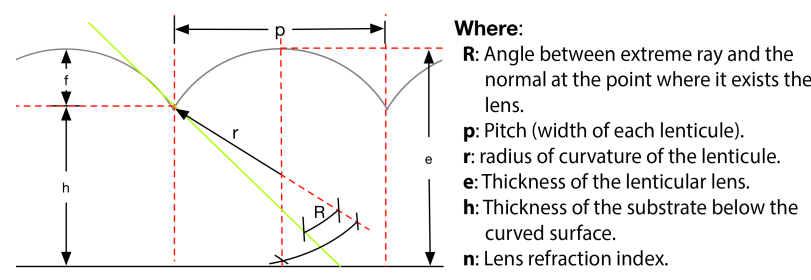

Figure 6: Detailed lenticular lens

discretize the light generated from each pixel and represent it as several rays that start from each subpixel and travel along the substrate of the lens and get refracted to the air from each lenticule. These rays are calculated in three steps: Substrate contact, lens contact and lens refraction.

\subsection{Step 1: Substrate contact.}

In this phase $n$ number of rays are calculated for each pixel with a spread $S$ (in deg) in order to get $n$ contact points $P_{0}, P_{1} \ldots P_{n}$ from a horizontal line (parallel to the screen) that defines the substrate thickness of the lens (fig. 7). To find the points $P_{0}, P_{1} \ldots P_{n}$ where the rays intersect the end of the lens substrate we represent the rays from the pixel and the substrate with line equations and find their respective intersection points. 


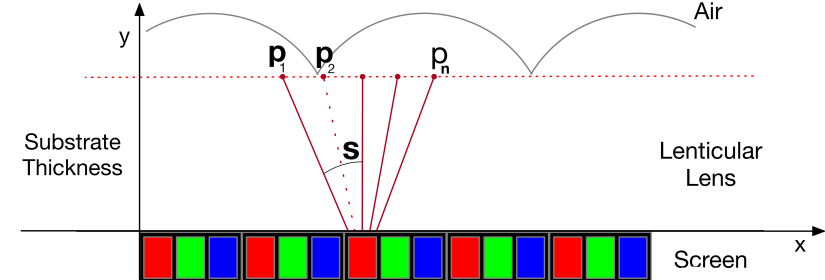

Figure 7: Step 1: Find where rays intersect substrate line.

Given the points $R_{1}$ and $R_{2}$ from the line $L_{1}$ that represents the ray that gets generated from the pixel and points $S_{1}$ and $S_{2}$ from the line $L_{2}$ that defines the substrate of the lens, we can generate two standard line equations with the form $y=m x+b$, make them equal on the $y$ axis (as it is the substrate thickness that the manufacturer gives) and find the intersection point $P_{i}$ on $x$ (fig. 7)

$$
\begin{aligned}
L_{1} \rightarrow y=m_{1} x+b_{1} & \quad L_{2} \rightarrow y=m_{2} x+b_{2} \\
m_{1} x+b_{1} & =m_{2} x+b_{2} \\
m_{1} x-m_{2} x & =b_{2}-b_{1} \\
x\left(m_{1}-m_{2}\right) & =b_{2}-b_{1} \\
x & =\frac{b_{2}-b_{1}}{m_{1}-m_{2}} \quad \text { intersection } \mathrm{X} \text { axis }\left(P_{x}\right) .
\end{aligned}
$$

Again, finding the $P_{y}$ becomes trivial as is given by the lens manufacturer and is the lens substrate thickness.

\subsection{Step 2: Lens contact.}

After finding where the rays of light intersect in the substrate thickness line, we proceed to find which lens the ray "belongs" to in order to apply the corresponding refraction in step three.

To do so, we find the center $C_{i}$ of the lenticule $l_{i}$ that is closest to the intersection $P_{j}$ in order to know which lens refracts each ray from the pixel (fig. 8). To find these centers we just need to find $C_{x}$ for each

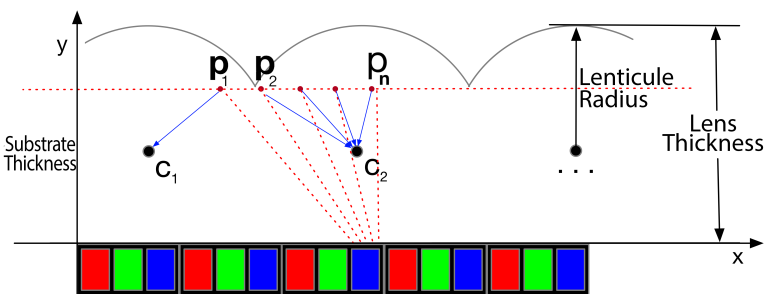

Figure 8: Step 2: Find points $P_{j}$ closest to center $C_{i}$.

lens because $C_{y}$ in all the lenticule centers remain the same and can be easily deduced from figure 8 as $C_{y}=$ lensThickness - lenticuleRadius.

Getting $C_{x}$ is also pretty straight forward as the pixel number (PixNum) the ray comes from is known, the physical pixel size ( $P p s)$ has already been precalculated from the screen density $P P I$ and we can know each lenticule size $(L s)$ by just dividing the LPI of the lens by 1 inch $\left(L s=\frac{1}{L P I}\right)$.

First, we calculate the lenticule center that stays on top of the current pixel (Lct) with:

$$
L c t=\left(\left\lfloor\frac{\text { PixNum } * \text { Pps }}{L s}\right\rfloor * L s\right)+\left(\frac{L s}{2}\right)
$$

After calculating Lct (eq. 1), we check if the distance with $P_{x} i$ is lesser than half of an individual lenticule size $\left(\left|L c t-P_{x}\right|<\frac{L s}{2}\right)$, if it is we have found the lenticule center $C_{x}$ the ray belongs to, else we carry checking for neighboor lenticule centers with $C_{x} \pm \frac{L s}{2}$ until the condition gets satisfied.

\subsection{Step 3: Lens Refraction.}

After figuring the closest lenticule center $C_{j}$ from a given ray intersection $P_{i}$ we can continue with the ray direction $\overrightarrow{\mathrm{r}}$ and finally find the intersection point $Q_{i}$ with the lenticule $L_{j}$ where the ray refraction occurs (fig. 9). $\overrightarrow{\mathrm{rf}}$

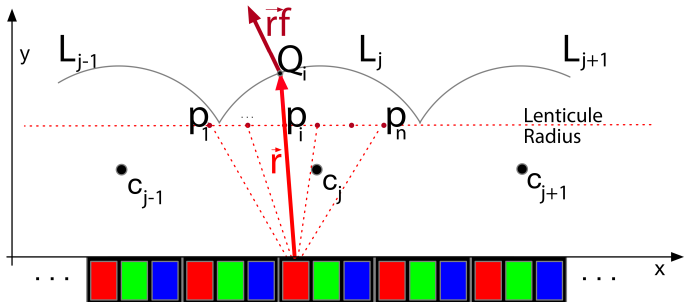

Figure 9: Step 3: Ray intersection with lens and refraction.

\subsubsection{Finding lens intersection point:}

To find $Q_{i}$ (fig 9) we can treat each lenticule as a circle and the rays that come from each pixel as lines and then the lens-ray intersection point can be treated as a linecircle intersection as follows [32] [33]: Given a circle with center $\left(c_{x}, c_{y}\right)$ with radius $r$ representing the lenticule with center $C_{j}$ and a line representing the ray of light that comes from a given pixel:

$$
\begin{gathered}
\quad \boldsymbol{R a y} \rightarrow y=m x+b \quad \text { Lens } \rightarrow\left(x-c_{x}\right)^{2}+\left(y-c_{y}\right)^{2}=r^{2} \\
0=\left(x-c_{x}\right)^{2}+\left(m x+\left(b-c_{y}\right)\right)^{2}-r^{2} \text { Replace ray in lens equation } \\
0=x^{2}\left(1+m^{2}\right)+x\left(2 m b-2 c_{x}-2 m c_{y}\right)+\left(c_{x}^{2}+b^{2}-2 b c_{y}+c_{y}^{2}-r^{2}\right)
\end{gathered}
$$

Solving the quadratic form of the resulting equation we end up with:

$$
\begin{aligned}
x_{1,2} & =\frac{-m b+c_{x}+m c_{y} \pm}{1+m^{2}} \\
& =\frac{\sqrt{-2 m b c_{x}+2 m c_{x} c_{y}-b^{2}+2 b c_{y}-c_{y}^{2}+r^{2}+r^{2} m^{2}-m^{2} c_{x}^{2}}}{1+m^{2}}
\end{aligned}
$$

After finding the $x$ component in eq. 2, there are three possible values that the quadratic equation gives us under the square root. Lets call this $D$.

$$
D=-2 m b c_{x}+2 m c_{x} c_{y}-b^{2}+2 b c_{y}-c_{y}^{2}+r^{2}+r^{2} m^{2}-m^{2} c_{x}^{2}
$$


If $D<0$ there is no intersection point, when $D=0$ the line touches the lens tangentially and finally if $D>0$ there are two intersection points (as each lenticule is at the end a circle.) We are only interested in the positive value as the lenses point torward the positive $Y$ axis so on this case, the $x$ component of the intersection point $Q_{i}$ where the ray touches the lens ends up being:

$$
\begin{aligned}
x & =\frac{-m b+c_{x}+m c_{y}+}{1+m^{2}} \\
& =\frac{\sqrt{-2 m b c_{x}+2 m c_{x} c_{y}-b^{2}+2 b c_{y}-c_{y}^{2}+r^{2}+r^{2} m^{2}-m^{2} c_{x}^{2}}}{1+m^{2}}
\end{aligned}
$$

Finally, by replacing eq. 3 on the line equation from the ray we can get the $y$ component of $Q_{i}$.

\subsubsection{Generating refracted ray from the lens:}

After finding the point of intersection where the ray (coming from the pixel) touches the lens $\left(Q_{i}\right)$ we finally calculate the refracted pixel ray $(\overrightarrow{r f})($ fig. 9) using Snell's law [34].

Snell's Law states that the products of the index of refraction and sines of the angles must be equal (eq. 4).

$$
n_{1} \sin \left(\theta_{1}\right)=n_{2} \sin \left(\theta_{2}\right)
$$

Snell's equation (eq. 4) can be re-written as:

$$
\sin \left(\theta_{2}\right)=\frac{n_{1}}{n_{2}} \sin \left(\theta_{1}\right)
$$

One can immediately see a problem here, and is that if $\sin \left(\theta_{1}\right)>\frac{n_{2}}{n_{1}}$ then $\sin \left(\theta_{2}\right)$ has to be bigger than 1 which is impossible. So when this happens, we have a TIR (Total Internal Reflection), TIR only happens if you go from a denser material (lens) to a less dense material (air). When TIR happens, we just ignore that ray and do nothing about it. So eq. 5 can be written like this:

$$
\sin \left(\theta_{2}\right)=\frac{n_{1}}{n_{2}} \sin \left(\theta_{1}\right) \longleftrightarrow \sin \left(\theta_{1}\right) \leq \frac{n_{2}}{n_{1}}
$$

To find $\overrightarrow{r f}$, lets begin by splitting it up in a tangent and a normal part:

$$
\overrightarrow{r f}=\overrightarrow{r f_{\|}}+r \vec{f}_{\perp}
$$

As all the vectors are normalized and any vector $\vec{v}$ can be decomposed in its tangent and parallel parts, and its parts are perpendicular to each other $\left(\overrightarrow{v_{\|}} \perp \overrightarrow{v_{\perp}}\right)$, with basic trigonometry, the following rules apply:

$$
\sin (\theta)=\frac{\left|\overrightarrow{v_{\|}}\right|}{|\vec{v}|}=\left|\overrightarrow{v_{\|}}\right| \quad \cos (\theta)=\frac{\left|\overrightarrow{v_{\perp}}\right|}{|\vec{v}|}=\left|\overrightarrow{v_{\perp}}\right|
$$

Since Snell's law talks about sines (eq. 6), we can use eq. 8 and rewrite eq. 6 as:

$$
\left|\overrightarrow{r f}_{\|}\right|=\frac{n_{1}}{n_{2}}\left|\vec{r}_{\|}\right|
$$

Since $\overrightarrow{r f} \|$ and $\vec{r}_{\|}$are parallel and point in the same direction, eq. 9 becomes:

$$
\overrightarrow{r f}_{\|}=\frac{n_{1}}{n_{2}} \vec{r}_{\|}=\frac{n_{1}}{n_{2}}\left(1-\cos \left(\theta_{r}\right) \vec{n}\right)
$$

To find $r \vec{f}_{\perp}$ one can simply use pythagoras $\left(|\vec{v}|^{2}=\right.$ $\left|\overrightarrow{v_{\|}}\right|^{2}+\left|\overrightarrow{v_{\perp}}\right|^{2}$ ) and end up with:

$$
r \vec{f}_{\perp}=-\sqrt{1-|\overrightarrow{r f}|^{2}} \vec{n}
$$

Replacing eq. 8 , eq. 9 and eq. 11 in eq. 7 we get:

$$
\overrightarrow{r f}=\frac{n_{1}}{n_{2}} \vec{r}-\left(\frac{n_{1}}{n_{2}} \cos \left(\theta_{r}\right)+\sqrt{1-\sin ^{2}\left(\theta_{r f}\right)}\right) \vec{n}
$$

Finally, we need to find $\sin ^{2}\left(\theta_{r f}\right)$ in this last equation, but this can be easily deduced it using Snell's law in equation 9 .

$$
\sin ^{2}\left(\theta_{r f}\right)=\left(\frac{n_{1}}{n_{2}}\right)^{2} \sin ^{2}\left(\theta_{r}\right)=\left(\frac{n 1}{n 2}\right)^{2}\left(1-\cos ^{2}\left(\theta_{r}\right)\right)
$$

With these two equations we can finally calculate the refracted vector $\overrightarrow{r f}$.

Each step is built with information from the previous step starting by disctretizing the light that comes from each pixel in rays, each ray is generated from the pixel until it intersects the lens substrate (step 1). Afterwards, we figure out which lenticule the ray "belongs to" and calculate the center of the aforementioned lenticule for the ray and get the position where the ray intersects with the lens (step 2). Finally, we calculate the new refracted ray for the ray that comes from the pixel (step 3). These three steps are performed for each pixel $n$ ray times for all the pixels in the screen.

\section{EXPERIMENT}

To assess how pixel spread correlates to the performance of lenticular lenses when multiplexing user views, two main factors need to be taken into account. The maximum ghosting perceived for each user and the minimum amount of unique pixels users perceive.

A simulation was performed to assess these factors. This experiment simulate separate lenses that can fit in 18 to 21 pixels per lenticule, lenses have a $50 \%$ radius increase from the minimum possible lenticule radius, lens substrate thickness of $0.1 \mathrm{~mm}$ with a refraction index of 1.56 (PETG)[8].

The aforementioned lens is designed to fit a Samsung QN65Q900R TV. This 8k 65" TV has a resolution of $7680 x 4320$ pixels, a refresh rate of $120 \mathrm{~Hz}$ with physical display dimensions of $1428.51 \times 803.538 \mathrm{~mm}$, pixel density of $137 \mathrm{ppi}$ and a pixel pitch of $0.186 \mathrm{~mm}$.

Tests where performed with three users evenly separated by 50 centimeters between them and 1 meter away from the screen as seen in figure 10 . 


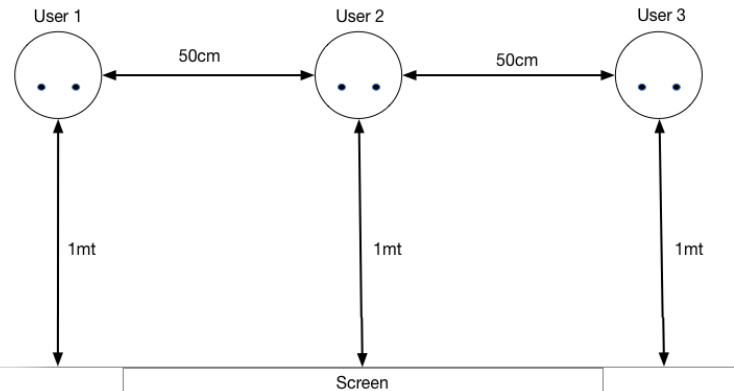

Figure 10: User separation diagram.

In this experiment we measure the minimum unique pixels perceived for each user and the maximum amount of image ghosting users get in said layout by varying the pixel spread, starting with a collimated light (0 deg spread) up to 89 degrees of pixel spread (fan like setting).

One thing to note is that in this paper we treat the spread angle as the angle from the center of the pixel to where the last ray ends (see figure 5). The total amount of "vision" from any given pixel would be twice the values of the reported spread here.

\section{DATA ANALYSIS}

Different spread angles will result in different metrics for ghosting and the amount of unique pixels perceived per user. As the reader can notice in table 1. As expected, the more angle spread pixel rays have the more ghosting appears between users. Also, in said table, the reader can see that regardless of the number of pixels per lenticule, the change between ghosting values is not representative.

The first thought that one could have after checking table 1 is that collimated light would perform best (as in there is no ghosting), but if the reader looks closely; with 10 degrees of pixel spread, ghosting just starts to appear. One can also see that when the pixel spread is above 20 degrees (measured from the center of the pixels), pretty much the ghosting occludes half of the imagery each user perceives.

Another thing to notice in table 1 is that ghosting increases drastically from 10 to 20 degrees and even more from 20 to 40 . For sure pixel spread needs to be below 20 degrees to have an usable optical routing approach when multiplexing images between users.

\begin{tabular}{|l|l|l|l|l|}
\hline $\begin{array}{l}\text { Spread } \\
\text { deg) }\end{array}$ & $\begin{array}{l}\text { Max } \\
\text { Ghosting } \\
18 \mathrm{px} / \mathrm{lent}(\%)\end{array}$ & $\begin{array}{l}\text { Max } \\
\text { Ghosting } \\
19 \mathrm{px} / \mathrm{lent}(\%)\end{array}$ & $\begin{array}{l}\text { Max } \\
\text { Ghosting } \\
\text { 20px/lent(\%) }\end{array}$ & $\begin{array}{l}\text { Max } \\
\text { Ghosting } \\
21 \mathrm{px} / \mathrm{lent}(\%)\end{array}$ \\
\hline \hline 0 & $0.000 \%$ & $0.000 \%$ & $0.000 \%$ & $0.000 \%$ \\
\hline 10 & $2.467 \%$ & $1.345 \%$ & $1.139 \%$ & $1.0566 \%$ \\
\hline 20 & $56.841 \%$ & $56.255 \%$ & $55.359 \%$ & $54.684 \%$ \\
\hline 30 & $85.607 \%$ & $85.580 \%$ & $85.070 \%$ & $84.974 \%$ \\
\hline 40 & $94.534 \%$ & $94.528 \%$ & $94.721 \%$ & $94.587 \%$ \\
\hline 50 & $94.630 \%$ & $94.603 \%$ & $94.567 \%$ & $94.466 \%$ \\
\hline 60 & $94.374 \%$ & $93.825 \%$ & $93.978 \%$ & $94.068 \%$ \\
\hline
\end{tabular}

Table 1: Maximum ghosting perceived between 18-21 pixels per lenticule.

In table 2 the reader can also appreaciate the minimum unique number of pixels perceived between either of the three users. As one can notice from 0 to 10 degrees of pixel spread, the minimum unique perceived pixels double up to $\sim 20 \%$ without generating pretty much any ghosting (as seen in table 1).

From 10 to 20 degrees (table 2) the minimum unique pixels also increase, but not as much as from 0 to 10 and unfortunately the ghosting (table 1) increases radically when reaching 20 degrees.

It is worth mentioning that with as little as $\sim 20 \%$ of unique pixels perceived from an $8 \mathrm{~K} \mathrm{TV}$, the resulting image will contain at least around 1500 pixels. Not bad for assembling an image.

\begin{tabular}{|l|l|l|l|l|}
\hline $\begin{array}{l}\text { Spread } \\
(\mathrm{deg})\end{array}$ & $\begin{array}{l}\text { Min Unique } \\
\text { seen pixels } \\
18 \mathrm{px} / \mathrm{lent}(\%)\end{array}$ & $\begin{array}{l}\text { Min Unique } \\
\text { seen pixels } \\
19 \mathrm{px} / \mathrm{lent}(\%)\end{array}$ & $\begin{array}{l}\text { Min Unique } \\
\text { seen Pixels } \\
20 \mathrm{px} / \mathrm{lent}(\%)\end{array}$ & $\begin{array}{l}\text { Min Unique } \\
\text { seen Pixels } \\
21 \mathrm{px} / \mathrm{lent}(\%)\end{array}$ \\
\hline \hline 0 & $12.291 \%$ & $12.072 \%$ & $11.875 \%$ & $11.718 \%$ \\
\hline 10 & $24.140 \%$ & $23.945 \%$ & $23.763 \%$ & $23.671 \%$ \\
\hline 20 & $32.526 \%$ & $32.356 \%$ & $32.291 \%$ & $32.760 \%$ \\
\hline 30 & $40.065 \%$ & $39.856 \%$ & $39.218 \%$ & $39.570 \%$ \\
\hline 40 & $46.093 \%$ & $45.820 \%$ & $45.625 \%$ & $45.338 \%$ \\
\hline 50 & $46.992 \%$ & $46.601 \%$ & $46.432 \%$ & $46.132 \%$ \\
\hline 60 & $46.458 \%$ & $46.054 \%$ & $45.898 \%$ & $45.820 \%$ \\
\hline
\end{tabular}

Table 2: Minimum unique perceived between 18-21 pixels per lenticule.

Analyzing the initial values from tables 1 and 2 begs to ask questions like: "How much can we push the pixel spread before introducing ghosting between users?" and also "How much more unique pixels are we gaining by increasing the spread angle before generating ghosting?".

In figure 11, the reader can see three aligned graphs, the first one shows the minimum percentage of the whole screen either of the users see, the second one presents the minimum amount of unique pixels users perceive and finally the third graph displays the maximum ghosting perceived by either of the aforementioned users.

As one can see, with a collimated light, the experiment generates zero ghosting but at the same time collimated 
light doesnt maximize the amount of perceived pixels per user. If one looks carefully on the second graph, the reader can see that as the pixel spread increases, still there is zero ghosting present in the third graph until reaching 9 degrees of pixel spread with around 1700 minimum unique perceived pixels.

Pixel Spread Report Samsung Q900R. Pixel size: 0.186004mm, Lens rad Incr: 50\% Resolution: 7680, Rays per pixel: 150, Substrate Thickness: $0.1 \mathrm{~mm}$ Min pixel percentage from 3 users (closest to $33 \%$ the better)

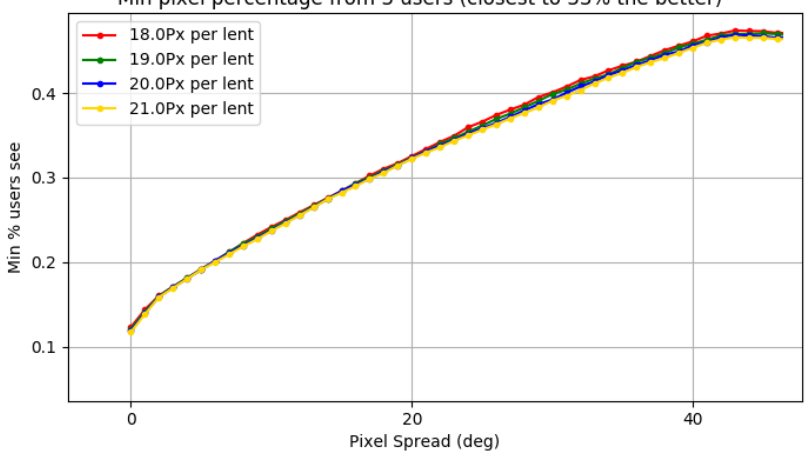
Minimum unique pixels from 3 users (the higher the better)

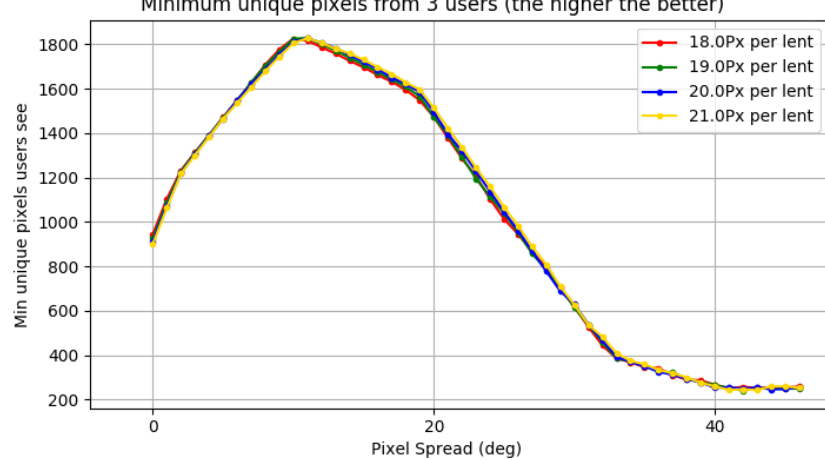

Max ghosting from 3 users (the lower the better)

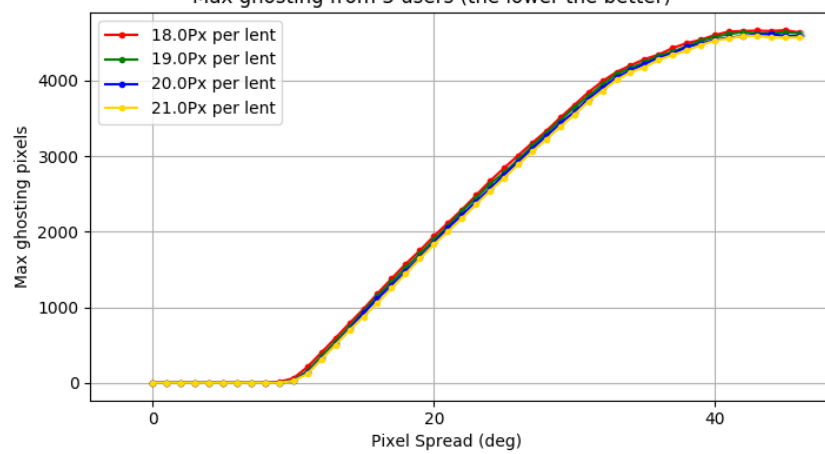

Figure 11: Pixel spread graph for Samsung Q900R 8k $65 " \mathrm{TV}$.

Looking more closely, unique perceived pixels get maximized when the pixel spread is 11 degrees but, at the same time ghosting starts to appear when the spread reaches 10 degrees. When the pixel spread is 11 degrees, the maximum amount of ghosting perceived by either of the users is $\sim 9 \%$.

Finally, in Figure 12 one can appreciate how ghosting also increases the thicker the substrate gets regardless the number of pixels per lenticule. With a substrate thickness of $0 \mathrm{~mm}$ (pretty much the lencitules directly attached to the TV), the ghosting is reduced the most.
Unfortunately no lenticular lens comes with a substrate thickness of $0 \mathrm{~mm}$. Hence the calculations done with a substrate thickness of $0.1 \mathrm{~mm}$.

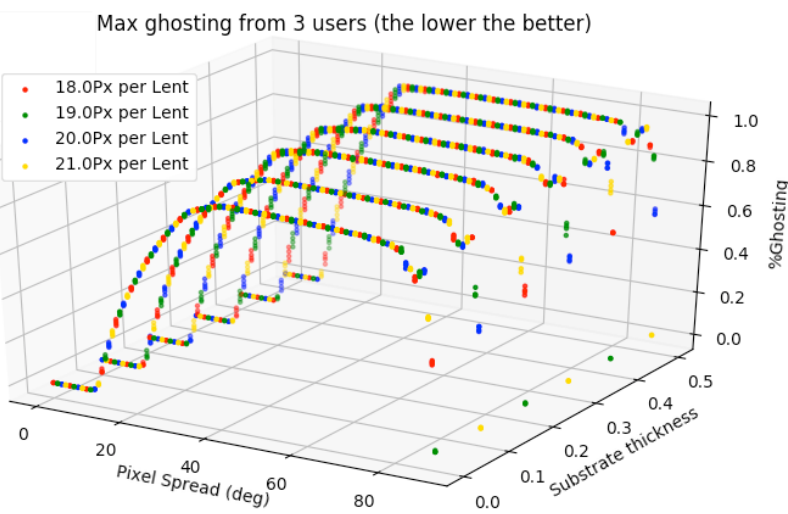

Figure 12: Ghosting vs Substrate thickness Samsung Q900R.

\section{CONCLUSIONS}

An overview of how lenticular lenses work and how are they used was presented in this paper; a lenticular lens simulator was also introduced and its internal mathematics on how it works where also exposed. An experiment with three users evenly separated to assess pixel spread on this paper was also mentioned and an analysis of the experiment was covered.

Ghosting is one of the biggest issues that deter the most user experience when multiplexing images through one screen. Pixel spread as discussed here is a contributing factor on ghosting when employing lenticular lenses. As presented in this paper the more straight the pixel rays the better in regards to reducing ghosting.

Lenticular lenses that have 18 to 21 pixels per lenticule, $50 \%$ of minimal lenticule radius increase and a substrate thickness of $0.1 \mathrm{~mm}$ behave similarly in regards of maximum amount of ghosting perceived and minimum amount of unique pixels displayed for either of the users.

The percentage of perceived ghosting radically increases from 10 to 20 degrees of pixel spread as seen in table 1. It is also clear that collimated pixel rays will generate zero ghosting, but will not maximize the minimum number of unique pixels perceived by either of the users as seen in figure 11 .

11 degrees of pixel spread maximizes the minimum amount of unique pixels perceived by the users but unfortunately introduces a perceived ghosting of $\sim 9 \%$. Nine degrees of pixel spread on the other hand almost maximizes the minimum unique perceived pixels by either of the users without introducing any ghosting to the final interlaced image in the proposed setup.

With a pixel spread of nine degrees, the minimum amount of unique pixels perceived is $\sim 23 \%$; more or 
less 1700 pixels. Enough to assemble an image that contains zero ghosting.

Given the case that it's not physically possible to manufacture a lens with said requirements of substrate thickness of $0.1 \mathrm{~mm}$; its feasible to increase the thickness to some extent without inducing ghosting with 9 degrees of pixel spread.

\section{FUTURE WORK}

Subpixel layout is something that changes from screen to screen; in this paper we covered a subpixel layout that follows the typical red/green/blue layout with even subpixel sizes. It would be interesting to see if the same pixel spread behavior repeats with different physical subpixel layouts.

The lenticular lens simulator presented in this paper helped assessing maximum ghosting and minimum amount of unique pixels perceived by either of the users; color banding is a phenomenom that occurs when lenticules magnify enough a pixel hence producing separate red/green/blue bands instead of an even white color. Color banding wasnt addressed in this paper and is worth doing more research on this topic to assess if the proposed lens with the current pixel sizes of the tested screen generates color banding.

The experiment proposed in this paper had static users; user movement should be considererd for the data in order to assess how much ghosting and pixel spread vary troughout the physical space. A mapping of the space would be valuable to manipulate where users should be located depending on the generated virtual content.

An interesting way to reduce pixel spread would be with privacy screens; said screens are cheap and easy to attach to a screen. It would be interesting to know if with the new material between the screen and the lens; color banding is produced or if ghosting and unique pixels change.

\section{REFERENCES}

[1] Zsolt Szalavári, Dieter Schmalstieg, Anton Fuhrmann, and Michael Gervautz. 'studierstube': An environment for collaboration in augmented reality. Virtual Reality, 3(1):37-48, 1998.

[2] Brice Pollock, Melissa Burton, Jonathan W Kelly, Stephen Gilbert, and Eliot Winer. The right view from the wrong location: Depth perception in stereoscopic multi-user virtual environments. IEEE Transactions on Visualization \& Computer Graphics, (4):581-588, 2012.

[3] Karen B Chen, Ryan A Kimmel, Aaron Bartholomew, Kevin Ponto, Michael L Gleicher, and Robert G Radwin. Manually locating physical and virtual reality objects. Human factors, 56(6):1163-1176, 2014.
[4] Mark Bolas, Ian McDowall, and Dan Corr. New research and explorations into multiuser immersive display systems. IEEE computer graphics and applications, 24(1):18-21, 2004.

[5] 3D Adworks. 3d lenticular printing. https://3dadworks.com/en/ 3d-lenticular-printing/, Mar 2018. Accessed: 2019-Apr-15.

[6] Zauberklang. Timeline of historical film colors. https://zauberklang.ch/ filmcolors/cat/lenticular-screen/ \#/. Accessed: 2019-Apr-15.

[7] Rachel Rosmarin. Give me 3d tv, without the glasses. https://www.tomsguide.com/ us / 3DTV-autostereoscopic-CES, review-1490.html, Jan 2010. Accessed: 2019-Apr-15.

[8] Juan Sebastian Munoz-Arango, Dirk Reiners, and Carolina Cruz-Neira. Maximizing lenticular lens performance for multi user vr displays. In Laval Virtual 2019, page 12. ACM, 2019.

[9] Microlens. Choosing the correct lenticular lens sheet. http://www.microlens.com/ pages/choosing_right_lens.htm, Nov 2018. Accessed: 2018-Nov-12.

[10] LenstarLenticular. Possible lenticular effects, Nov 2018 https://www.lenstarlenticular.com/lenticulareffects/ Accessed: 2018-Nov-12.

[11] Gordon R Little, Steven C Gustafson, and Vasiliki E Nikolaou. Multiperspective autostereoscopic display. In Cockpit Displays, volume 2219, pages 388-395. International Society for Optics and Photonics, 1994.

[12] Cees Van Berkel and John A Clarke. Characterization and optimization of 3d-lcd module design. In Stereoscopic Displays and Virtual Reality Systems IV, volume 3012, pages 179-187. International Society for Optics and Photonics, 1997.

[13] Cees Van Berkel. Image preparation for $3 \mathrm{~d}$ lcd. In Stereoscopic Displays and Virtual Reality Systems VI, volume 3639, pages 84-92. International Society for Optics and Photonics, 1999.

[14] Kenji Matsumoto and Toshio Honda. Research of 3d display using anamorphic optics. In Stereoscopic Displays and Virtual Reality Systems IV, volume 3012, pages 199-208. International Society for Optics and Photonics, 1997.

[15] Katsuyuki Omura, Shinichi Shiwa, and Tsutomu Miyasato. Lenticular autostereoscopic display system: multiple images for multiple viewers. Journal of the Society for Information Display, 6(4):313-324, 1998. 
[16] Lenny Lipton and Mark H Feldman. New autostereoscopic display technology: the synthagram. In Stereoscopic Displays and Virtual Reality Systems IX, volume 4660, pages 229-236. International Society for Optics and Photonics, 2002.

[17] Wojciech Matusik and Hanspeter Pfister. 3d tv: a scalable system for real-time acquisition, transmission, and autostereoscopic display of dynamic scenes. In ACM Transactions on Graphics (TOG), volume 23, pages 814-824. ACM, 2004.

[18] David Nguyen and John Canny. Multiview: spatially faithful group video conferencing. In Proceedings of the SIGCHI conference on human factors in computing systems, pages 799-808. ACM, 2005.

[19] David T Nguyen and John Canny. Multiview: improving trust in group video conferencing through spatial faithfulness. In Proceedings of the SIGCHI conference on Human factors in computing systems, pages 1465-1474. ACM, 2007.

[20] Yasuhiro Takaki. Thin-type natural threedimensional display with 72 directional images. In Stereoscopic Displays and Virtual Reality Systems XII, volume 5664, page 56. International Society for Optics and Photonics, 2005.

[21] Hiroshi Nakanuma, Hiroyuki Kamei, and Yasuhiro Takaki. Natural 3d display with 128 directional images used for human-engineering evaluation. In Stereoscopic Displays and Virtual Reality Systems XII, volume 5664, pages 28-36. International Society for Optics and Photonics, 2005.

[22] Kengo Kikuta and Yasuhiro Takaki. Development of svga resolution 128-directional display. In Stereoscopic Displays and Virtual Reality Systems XIV, volume 6490, page 64900U. International Society for Optics and Photonics, 2007.

[23] Yasuhiro Takaki. Super multi-view display with 128 viewpoints and viewpoint formation. In Stereoscopic Displays and Applications XX, volume 7237, page 72371T. International Society for Optics and Photonics, 2009.

[24] Yasuhiro Takaki, Osamu Yokoyama, and Goro Hamagishi. Flat panel display with slanted pixel arrangement for 16-view display. In Stereoscopic Displays and Applications XX, volume 7237, page 723708. International Society for Optics and Photonics, 2009.

[25] Yasuhiro Takaki and Nichiyo Nago. Multiprojection of lenticular displays to construct a 256-view super multi-view display. Optics express, 18(9):8824-8835, 2010.

[26] Phil Surman, Ian Sexton, Klaus Hopf, Wing Kai Lee, Richard Bates, Wijnand IJsselsteijn, and Ed- ward Buckley. Head tracked single and multi-user autostereoscopic displays. 2006.

[27] Rajwinder Singh Brar, Phil Surman, Ian Sexton, Richard Bates, Wing Kai Lee, Klaus Hopf, Frank Neumann, Sally E Day, and Eero Willman. Laserbased head-tracked 3d display research. Journal of Display Technology, 6(10):531-543, 2010.

[28] Rajwinder Singh Brar, Phil Surman, Ian Sexton, and Klaus Hopf. Multi-user glasses free 3d display using an optical array. In 3DTV-Conference: The True Vision-Capture, Transmission and Display of $3 D$ Video (3DTV-CON), 2010, pages 1-4. IEEE, 2010.

[29] Robert Kooima, Andrew Prudhomme, Jurgen Schulze, Daniel Sandin, and Thomas DeFanti. A multi-viewer tiled autostereoscopic virtual reality display. In proceedings of the 17th ACM Symposium on Virtual Reality Software and Technology, pages 171-174. ACM, 2010.

[30] Alioscopy about us. urlhttp://www.alioscopy.com/en/about_us.php. Accessed: 2019-Apr-15.

[31] Shang-Fei Zang, Qiong-Hua Wang, Wu-Xiang Zhao, Jie Zhang, and Jing-Long Liang. A frontal multi-projection autostereoscopic $3 \mathrm{~d}$ display based on a 3d-image-guided screen. Journal of display technology, 10(10):882-886, 2014.

[32] AmBrSoft. Intersection of a circle and a line. http://www.ambrsoft.com/ TrigoCalc/Circles2/circlrLine_ . htm, Feb 2018. Accessed: 2018-Nov-14.

[33] Attila's Projects. Circle and line intersection. http://apetrilla. blogspot.com/2011/12/ circle-and-line-intersection. html, Dec 2011. Accessed: 2018-Nov-14.

[34] Bram De Greve. Reflections and refractions in ray tracing. Retrived Oct, 16:2014, 2006. 